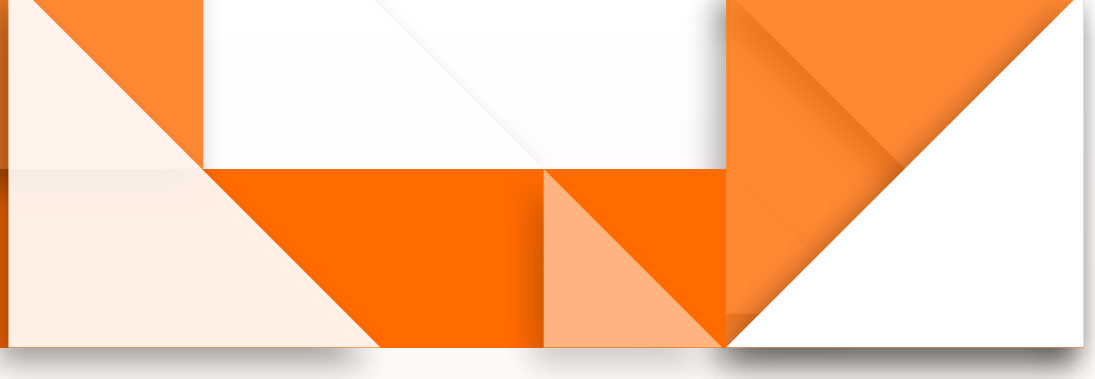

\title{
Ecología para la formación inicial de profesores a partir de los affordances de las TIC
}

\section{- Ecology for Early Teacher Training Based on Affordances of ICT}

- Ecologia para a formação inicial de professores a partir dos affordances das TIC

\section{Resumen}

Este artículo presenta una reflexión en torno al desarrollo de competencias para la integración de las tecnologías de la información y la comunicación (TIC) en la educación durante la formación inicial de profesores. En el documento, se analizan específicamente el conjunto de políticas, estándares y estrategias construidas con este propósito. A partir de los avances alcanzados y de la caracterización de las dinámicas de producción de conocimiento en la sociedad contemporánea, surge la pregunta por las posibilidades que las TIC en sí mismas pueden proveer a la formación inicial de profesores en el desarrollo de sus competencias para integrarlas en la educación. Por otro lado, también se indaga si las nuevas tendencias de aprendizaje, materializadas en los conceptos de affordance y ecologías del aprendizaje, pueden convertirse en rutas apropiadas para afianzar su desarrollo. Se considera que una perspectiva ecológica para el aprendizaje, construida a partir de los affordances o potencialidades de las tic, puede fortalecer la integración de la relación entre educación y tecnología en la formación docente, que hasta la fecha se ha pensado de manera independiente y desarticulada.

Palabras clave

ecologías de aprendizaje; formación inicial de profesores; políticas y estándares; tecnología educativa

\section{Linda Alejandra Leal-Urueña* Julio Ernesto Rojas-Mesa*}

Magíster en Tecnologías de Información Aplicadas a la Educación, profesora asistente del Departamento de Tecnología, Universidad Pedagógica Nacional, Bogotá, Colombia. Estudiante del Doctorado en Educación, Universidad Nacional de Educación a Distancia UNED.

lalealu@pedagogica.edu.co,

ORCID: http://orcid.org/0000-0002-8245-1096

** Doctor en Teoría de la Educación y Pedagogía Social, profesor del Doctorado en Educación, Universidad Santo Tomás, Bogotá, Colombia.

juliorojas@usantotomas.edu.co.

ORCID: http://orcid.org/0000-0002- 2237-3727 


\begin{abstract}
In this paper, we reflect on the development of competences for the integration of information and communication technologies (ICTS) in education during early teacher training. Specifically, we analyze the set of policies, standards and strategies built for this purpose. From the progress made and the characterization of the dynamics of knowledge production in contemporary society, the question arises about the possibilities that ICTS can provide the initial training of teachers in the development of their skills to integrate them in education. We also investigate whether the new learning trends, materialized in the concepts of affordance and ecologies of learning, can become appropriate ways to strengthen their development. It is believed that an ecological perspective for learning, built from the affordances or potentialities of icts, can strengthen the integration of the relationship between education and technology in teacher training, which so far has been thought of in an independent and disjointed way.

Keywords

learning ecologies; preservice teacher education; policies and standars; educational technology

\section{Resumo}

Este artigo apresenta uma reflexão sobre o desenvolvimento de competências para a integração das tecnologias da informação e comunicação (TIC) na educação durante a formação inicial de professores. No documento, analisam-se especificamente o conjunto de políticas, estândares e estratégias construídas com esse propósito. A partir dos avanços conseguidos e da caracterização das dinâmicas de produção de conhecimento na sociedade contemporânea, surge a questão pelas possibilidades que as TIC em si mesmas podem dar à formação inicial de professores no desenvolvimento de suas competências para integrá-las na educação. Por outro lado, também se questiona se as novas tendências da aprendizagem, materializadas nos conceitos de affordance e ecologias da aprendizagem, podem virar rotas apropriadas para consolidar seu desenvolvimento. Considera-se que uma perspectiva ecológica para a aprendizagem, construída a partir dos affordances ou potencialidades das TIC, pode fortalecer a integração da relação entre educação e tecnologia na formação docente, que até hoje foi pensada de forma independente e desvinculada.
\end{abstract}

Palavras-chave

alfabetização informacional; competências do professor; ecologias da aprendizagem; formação preparatória de professores; tecnologia educacional 


\section{Introducción}

Esta reflexión se orienta por varias preguntas. La primera de ellas es ¿̇cuáles son los avances en materia de construcción de políticas y estándares en torno al desarrollo de competencias TIC en la formación de docentes? La respuesta a este interrogante posibilita entender de manera contextual la exploración que se deriva de la segunda pregunta: las estrategias implementadas en la actualidad, durante la formación inicial de profesores, para desarrollar sus competencias de integración de TIC Żresponden efectivamente a las nuevas formas de aprendizaje en la era digital? Por último, y fruto de la reflexión generada, el artículo se desarrolla con una pregunta enfocada hacia una posible ruta para desplegar en el mundo de los docentes en formación un tema bastante discutido pero con poca instalación de significados en el aula: ¿̇de qué forma pueden aprovecharse los affordances o potencialidades de las TIC para actualizar y hacer más pertinentes los procesos de formación inicial de profesores en el desarrollo de sus competencias para integrar las TIC en la educación?

Para abordar la reflexión en torno a estos interrogantes se presenta un panorama general de las políticas para la formación inicial de profesores en TIC en América Latina y en Colombia, se analizan algunos de los estándares internacionales de formación de competencias digitales para profesores y se exponen los avances alcanzados por la investigación en el campo de las estrategias para el desarrollo de competencias para la integración de las TIC en la educación durante la formación inicial de profesores.

Enseguida, se presentan los retos y desafíos que afrontan las instituciones y programas de formación inicial de profesores de cara a las necesidades y demandas de la sociedad contemporánea sobre el rol de los futuros profesores en la apropiación de las TIC para el desarrollo de las competencias digitales de los estudiantes, la alfabetización mediática e informacional de los ciudadanos del siglo XXI, así como en el aprovechamiento de los avances tecnológicos disponibles para el desarrollo de habilidades fundamentales, como la capacidad de innovación, el pensamiento crítico, la resolución de problemas, la comunicación, la investigación y las competencias específicas de los dominios de conocimiento. La reflexión se aproxima a los desafíos de los programas de formación inicial de profesores, especialmente los relacionados con la necesidad de asumir un enfoque integrador de la tecnología, la pedagogía y los conocimientos disciplinares, así como la importancia de integrar diferentes affordances de las TIC con el propósito de fortalecer la efectividad, innovación y pertinencia del proceso de formación de los futuros profesores.

\section{Tesis y argumentos}

\section{Las políticas de formación inicial de} profesores en TIC en América Latina

De acuerdo con los análisis realizados por Vaillant (2013) en torno a la política de integración de TIC en los programas de formación de profesores en América Latina, existe un consenso en cuanto a que esta se ha pensado y formulado en estrecha relación con los Planes de Acción sobre la Sociedad de la Información y del Conocimiento de América Latina y el Caribe elAC $(2007,2010,2015,2018)$ de la Comisión Económica para América Latina y el Caribe (Cepal) y el programa Metas 2021, formulado por la Organización de Estados Iberoamericanos (OEI) y la Cepal. Por su parte, Brun (2011) destaca además en este contexto 
la importancia de los Objetivos del Milenio fijados en el año 2000 por los 189 países miembros de las Naciones Unidas.

El programa Metas 2021 surgió como un proyecto colectivo iberoamericano orientado a promover la cooperación educativa para el logro, entre otros objetivos, de la provisión de equipamiento tecnológico, la incorporación de las TIC en el currículo con sentido pedagógico y la generación de competencias en los estudiantes para aprender a aprender, asimismo, de formar a los profesores para que desarrollen las competencias necesarias que les permitan incorporar de forma natural las TIC en su práctica pedagógica como "variable fundamental para garantizar el éxito del esfuerzo emprendido" (OEl, 2010, p. 116). Sin embargo, ninguna de las metas menciona de manera explícita el uso y formación en competencias TIC durante la preparación inicial de profesores (Brun, 2011) y, en este marco, se constituye en un desafío de vital importancia para una educación de calidad.

Recientemente, la Agenda ELAC 2018 plantea el uso de tecnologías digitales como instrumentos para el desarrollo sostenible, promueve la creación del ecosistema digital en América Latina y el Caribe, mediante la integración y cooperación regional, y el fortalecimiento de las políticas digitales que impulsen el conocimiento, la inclusión, la equidad, la innovación y la sostenibilidad ambiental. Como propósito del área temática de Desarrollo Sostenible e Inclusión de esta agenda se ha establecido fortalecer el uso de las TIC en la educación. Más allá de un marco de referencia para el diseño de las políticas nacionales en la región, esta agenda se constituye en un escenario de cooperación para su implementación (Cepal, 2018).

En síntesis, a lo largo de la última década, en algunos países de la región se han elaborado documentos marco de política nacional para la integración de las TIC al sistema educativo, pero solo en algunos de ellos se hace referencia a la formación inicial docente. Es el caso de los documentos ministeriales de Chile en 2005, Brasil en 2009, Paraguay en 2009 y Panamá en 2010 (Vaillant, 2013).

\section{La "micropolítica institucional" de integración de las TIC: un vacío de gestión}

Es importante resaltar que en este contexto de macropolítica, desde el otro extremo, es decir desde la micropolítica, las instituciones encargadas de la formación inicial de profesores en la región aún no fortalecen propuestas de política institucional para la integración de las TIC en su oferta educativa. En el análisis de esta problemática se ha identificado una debilidad general de la política de las instituciones formadoras de maestros sobre la preparación de los nuevos docentes para el uso de las TIC con propósitos educativos:

La formación para el uso pedagógico de las TiC en la formación inicial de los docentes es extremadamente débil en casi todos los países de la región. Ellos han enfocado en su mayoría la capacitación básica en el área de TIC en la formación 
en servicio, pero con una baja cobertura.

Sólo seis países tienen programas a nivel nacional y todos registran cifras inferiores al

$50 \%$ de los docentes capacitados en el uso de las TIC. (Orealc/Unesco, 2016, p. 18).

Algunos planes de formación de futuros profesores aún no contemplan el estudio de las TIC y sus usos e impactos en los procesos de enseñanza-aprendizaje. Otros incluyen asignaturas específicas sobre el uso de las TIC en educación, mientras que en otros se plantea que estas competencias se deben adquirir de forma transversal en el conjunto de las asignaturas del plan de estudios.

De acuerdo con Orealc/Unesco "los centros de formación de profesores, salvo escasas excepciones, no han integrado adecuadamente el uso de las tecnologías en la formación de los futuros docentes" (2016, p. 6). En este mismo sentido, el informe de Siteal (2014) advertía que la formación en este campo no ha sido relevante para las instituciones formadoras de maestros. Ese retraso, o falta de atención no es un asunto menor, considerando que la formación inicial docente es un asunto estratégico para el aprovechamiento de la tecnología en las escuelas.

\section{Estándares para la formación inicial de competencias TIC en los profesores}

En materia de estándares para el fortalecimiento de competencias digitales en la integración de TIC en la educación, los más relevantes son: el Marco común de competencia digital docenteEspaña (Intef, 2017); ISTE-USA (ISTE, 2016); Estructura de competencias TIC para profesores (Unesco, 2011); Alfabetización mediática e informacional de Unesco (Wilson, Grizzle, Tuazo, Akyempong y Cheung, 2011); y las Competencias TIC para el desarrollo profesional docente del Ministerio de Educación de Colombia (MEN, 2013).

Estos estándares proponen una amplia gama de competencias digitales para la formación inicial de profesores, que pueden analizarse en cuatro categorías: (1) competencias generales de uso de las TIC con propósitos de información, interacción y creación de contenidos; (2) competencias para el aprovechamiento del potencial pedagógico de las tecnologías para potenciar y facilitar el aprendizaje y la construcción de conocimiento; (3) competencias para la formación de ciudadanía digital; y (4) competencias de TIC para la gestión y evaluación (véase la tabla 1).

Tabla 1. Estándares de formación inicial de competencias TIC para profesores

\begin{tabular}{|c|c|c|c|c|c|c|c|c|c|c|}
\hline $\begin{array}{c}\text { Categorías de } \\
\text { competencias } \\
\text { Estándar }\end{array}$ & 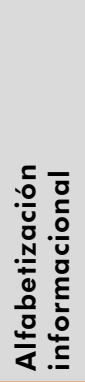 & 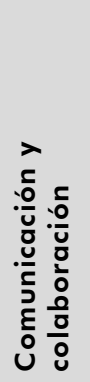 & 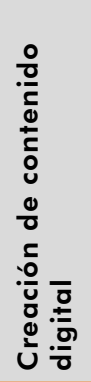 & 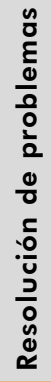 & $\begin{array}{l}\frac{0}{0} \\
\frac{0}{0} \\
\frac{0}{0} \\
\frac{i}{0} \\
. \frac{0}{0} \\
\frac{N}{0} \\
\frac{c}{0} \\
\frac{0}{2}\end{array}$ & 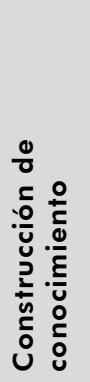 & 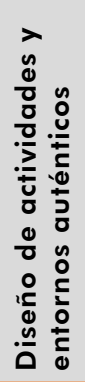 & 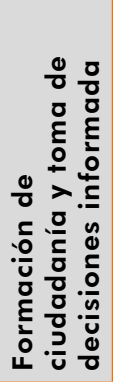 & 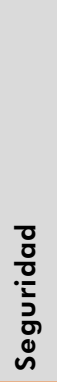 & 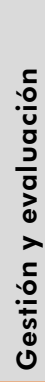 \\
\hline Intef (2017) & $x$ & $x$ & $x$ & $x$ & & & & & $x$ & \\
\hline ISTE (2016) & & $x$ & & $\mathrm{x}$ & $x$ & & $x$ & $x$ & & \\
\hline Unesco TIC (2011) & & & & $\mathrm{x}$ & $x$ & $x$ & & & & \\
\hline Unesco Mil (2011) & $x$ & & & $x$ & & & & $x$ & $x$ & \\
\hline MEN Colombia (2013) & & $x$ & & $x$ & $x$ & $x$ & & & & $x$ \\
\hline
\end{tabular}

Fuente: elaboración propia. 
Como se aprecia en la tabla 1, la competencia para integrar las TIC en la resolución de problemas es un tema recurrente en todos los estándares y representa el interés por avanzar en la apropiación de estas como vehículo para profundizar en el conocimiento y su aplicación en la solución de problemas reales. Otros dos temas que aparecen en al menos tres de las propuestas de estándares son: (1) las competencias para aprovechar las TIC con el fin de potenciar el aprendizaje efectivo y permanente en los estudiantes, proceso en el cual el profesor es un actor fundamental como facilitador y activador de las indagaciones que realiza el estudiante; y (2) las competencias para comunicarse y colaborar usando las tic.

En el estándar propuesto por el MEN (2013), se suma a las tres competencias mencionadas en los estándares internacionales, la aplicación de las TIC para la construcción de conocimiento, en concordancia con los planteamientos del estándar TIC de la Unesco. Además, se plantean las competencias relacionadas con la gestión y evaluación educativa e institucional, que no aparecen en ninguno de los estándares internacionales analizados, pero resultan pertinentes en el contexto colombiano debido a los rezagos en la implantación de sistemas de información, gestión y toma de decisiones en las instituciones educativas.

\section{La formación inicial de profesores en TIC en las políticas públicas de Colombia}

La integración de las TIC en el sistema educativo colombiano fue enunciada por la ley general de educación (1994) mediante la inclusión de la tecnología y la informática como objeto de estudio y como una de las nueve áreas obligatorias de la educación básica. Desde entonces, diversas leyes y decretos han configurado la Estrategia Nacional de Ciencia, Tecnología e Innovación, dentro de la cual la educación y las TIC desempeñan un papel principal.

A lo largo de casi tres décadas se ha creado el marco jurídico para la ejecución de una estrategia nacional de uso de TIC en educación (Galvis, 2014). Entre las políticas más recientes se encuentran: el Plan Decenal de Educación 20162026 (MEN, 2017), el Plan Nacional de Desarrollo 2014-2018 (Departamento Nacional de Planeación, DNP, 2015), el Plan Nacional de tIC 2008-2019 (Ministerio de Comunicaciones, 2008), la creación de la Oficina de Innovación Educativa con uso de NTIC (MEN, 2009) y los lineamientos del Ministerio de Educación Nacional Competencias TIC para el desarrollo profesional docente (MEN, 2013). Las iniciativas para el uso de TIC en educación también han formado parte de los planes de gobierno desde la década de los noventa. En el periodo comprendido entre 1990 y 2002 se crearon y estructuraron iniciativas para integrar la informática educativa y de los medios audiovisuales a los procesos educativos; durante el periodo 2006-2010 se institucionalizó en el Programa Nacional de Uso de Medios y de TIC; y en el actual periodo 2010-2018 se busca consolidar el Sistema Nacional de Innovación Educativa con uso de TIC. Al tenor de la política nacional, el men desarrolló simultáneamente múltiples programas 
de formación de competencias TIC dirigidos a profesores en ejercicio durante el periodo 2006-2016 (Galvis, 2014).

Las políticas públicas en esta materia también incluyen los planes decenales de educación. El más reciente (2016-2026), dedica su sexto desafío a la educación y las TIC enunciado de la siguiente manera: "Impulsar el uso pertinente, pedagógico y generalizado de las tecnologías para apoyar la enseñanza, la construcción de conocimiento, el aprendizaje, la investigación y la innovación, fortaleciendo el desarrollo para la vida" (MEN, 2017a, p. 17) y define entre sus lineamientos la formación de los maestros en el uso pedagógico de las diversas tecnologías y el fomento al uso de las TIC y de diversas tecnologías que impacten en el aprendizaje y promoción de las competencias del estudiante en el siglo xxI. Específicamente formula doce lineamientos estratégicos desde la formación docente para el uso pertinente y generalizado de las TIC (MEN, 2017a).

Además, el MEN (2017b) reglamentó los criterios de calidad para la formación inicial de profesores. Entre ellos se incluye la apropiación y uso pedagógico de las tic, en el componente de fundamentos generales, y la capacidad para incorporar su uso con criterio pedagógico en los procesos educativos en su contexto sociocultural, en el componente de didáctica de las disciplinas de los planes de estudio.

La especificidad de los lineamientos de política formulados por el MEN sobre la formación de competencias para la integración de las TIC en la educación, desde la formación inicial de los profesores, refleja la urgente necesidad de asegurar la actualización de los planes de estudios de los programas de formación de maestros como escenario natural para la preparación y desarrollo de las competencias y condiciones que requiere el ejercicio docente en la sociedad colombiana en la época contemporánea.
Respecto a las políticas públicas en Colombia es importante concluir que, en primer lugar, ha habido una intensa actividad desde las iniciativas gubernamentales en torno a la inserción de las TIC, en muchos casos como respuesta a diferentes momentos de la política internacional. En segundo lugar, se resalta la ausencia de liderazgo, en términos de política institucional, por parte de las instituciones de educación superior para asumir planes y estrategias de formación con impacto local, regional y nacional. Aún brillan por su ausencia las iniciativas de planeación educativa que marquen un cambio significativo en la integración de TIC en el proceso de formación inicial docente.

\section{Estrategias de formación inicial de profesores para integrar las TIC en la educación}

El segundo interrogante que se ha formulado como eje de esta reflexión consiste en la indagación acerca de si las estrategias de formación inicial de profesores implementadas en la actualidad corresponden efectivamente al desarrollo de competencias de integración de las TIC en la educación durante dicho proceso y se encuentran actualizadas con las nuevas formas de aprendizaje en la era digital.

En este sentido se presentan cinco tipos de estrategias reseñadas en las investigaciones recientes: (1) aprendizaje vicario, (2) aprendizaje activo y basado en el diseño y producción de artefactos de tecnología educativa, (3) trabajo colaborativo entre pares, (4) experiencias de integración de tecnología en entornos y situaciones de trabajo reales y (5) retroalimentación continua durante el proceso de formación.

El aprendizaje vicario es una de las estrategias más referenciadas. En él, los educadores de profesores sirven como modelos del uso de las TIC con propósito educativos. La experiencia en el uso de la tecnología que 
adquieren los docentes durante su formación, tanto por su propio uso como por el que hacen sus profesores, es un factor crucial para el desarrollo de esta competencia (Tondeur, Pareja Roblin, van Braak, Voogt y Prestridge, 2017). Sin embargo, las investigaciones advierten que la incidencia de los modelos es de doble vía: puede ser positiva, si las prácticas observadas resultan exitosas, o por el contrario puede incrementar los temores hacia el uso de las tecnologías si durante la observación se presentan dificultades (Instefjord y Munthe, 2017).

Asimismo, las experiencias de aprendizaje vicario definen la perspectiva de uso de la tecnología. La evidencia al respecto revela que en dichas experiencias aún predominan enfoques de aprendizaje estructurado con pocas oportunidades para el uso de tecnología centrada en el estudiante (Tondeur et al., 2017) y la transferencia de contenidos más que su uso creativo (Fullan y Langworthy, 2014).

El aprendizaje activo y basado en el diseño y producción de artefactos de tecnología educativa parte del principio de que los profesores en formación deben ser más que usuarios pasivos o consumidores de tecnología con fines demostrativos y deben asumir un rol activo y constructivo como diseñadores de tecnología educativa (Chien, Chang, Yeh y Chang, 2012). De esta manera, durante su formación inicial los profesores deben tener la oportunidad de diseñar y construir actividades, materiales educativos y espacios de aprendizaje integrando las tic. Cuando participan en el diseño e implementación colaborativa de soluciones educativas, trayectorias de aprendizaje y lecciones que involucran el uso de las tic, surgen y se integran sus conocimientos tecnológicos, pedagógicos y de contenido (Ling y Chai, 2016; Niess, 2017).

El análisis de esta estrategia desde la perspectiva sociocultural nos muestra que la construcción de artefactos es central en los procesos de aprendizaje y producción de conocimiento, no solo por la íntima conexión entre el pensamiento de los estudiantes y los artefactos que ellos utilizan y producen, sino por la importancia de comprender el aprendizaje a partir de la interacción entre estudiantes, profesores y artefactos (Olsson y Edman-Stålbrant, 2008) y entre sistemas culturales, educativos y tecnológicos (Rojas-Mesa y Leal-Urueña, 2017). La interacción entre los individuos no sucede solo a través de palabras y conceptos, sino mediante el desarrollo de "objetos" compartidos (artefactos y prácticas) y de sus iteraciones (Paavola y Hakkarainen, 2009). La construcción de conocimiento involucra centralmente la creación y refinamiento de artefactos, que encarnan el conocimiento como objetos físicos o virtuales (Scardamalia y Bereiter, 2014). Esta visión coincide con las teorías constructivistas del aprendizaje que argumentan que los aprendices construyen conocimiento activamente, en especial cuando están comprometidos construyendo objetos (Kafai, 1995; Li, 2012 y Papert, 1980), y es consistente con el creciente reconocimiento del rol del profesor como diseñador de tecnología educativa (Kali, McKenney y Sage, 2015) (McKenney, Kali, Markauskaite y Voogt, 2015) y de la educación como ciencia de diseño (Voogt, Fisser, Tondeur y van Braak, 2016). 
En tercer lugar, se encuentra la estrategia que agrupa las experiencias de formación sobre trabajo colaborativo. Sus beneficios incluyen: compartir experticia, facilitar la ayuda mutua, obtener mejores productos como consecuencia de la continua retroalimentación, el uso efectivo del tiempo dentro y fuera del aula, un alto nivel de desafío, mitigación de los temores de los profesores en formación cuando diseñan materiales de aprendizaje haciendo uso de las TIC (Tondeur et al., 2012). Las estrategias de trabajo en equipo pueden incluir diferentes configuraciones de grupo teniendo en cuenta el perfil de desempeño tecnológico de los estudiantes, lo cual les permite compartir sus habilidades y complementarlas (Tondeur, Scherer, Siddiq y Baran, 2017).

La promoción de experiencias de integración de tecnología en entornos y situaciones de trabajo reales durante el proceso formativo permiten comprender el contexto en el que tiene lugar el proceso educativo y prepararse para usar las TIC de diversas maneras, de acuerdo con las especificidades de los entornos educativos y aprovechando la infraestructura tecnológica disponible en las instituciones (Tondeur et al., 2012). Por otro lado, estas vivencias generan un fuerte sentido de logro; permiten aplicar el conocimiento pedagógico, tecnológico y de contenido durante la planeación, preparación y desarrollo de las intervenciones; y proporcionan a los potenciales profesores experiencias concretas para crear y aplicar sus conocimientos en el mundo real.

Finalmente, la estrategia de retroalimentación continua durante el proceso de formación enriquece el proceso de aproximación y diseño de situaciones de aprendizaje con tecnologías (Tondeur, Braak, Sang, Voogt, Fisser y Ottenbreit-Leftwich, 2012). La participación en experiencias formativas en las que los profesores en formación son sujetos de evaluación, colaboración y aplicación de los conocimientos a la solución de problemas y a la construcción de artefactos de tecnología educativa, facilita el que se involucren en procesos de retroalimentación recursiva como parte integral de la experiencia de aprendizaje. Estas prácticas contribuyen a profundizar el aprendizaje, clarificar ideas, identificar los vacíos en las producciones de conocimiento, mejorar el razonamiento y solucionar problemas (Smith, McCarthey y Magnifico, 2017).

\section{Nuevas aproximaciones necesarias para abordar la formación inicial de profesores en la sociedad contemporánea}

En un entorno caracterizado por el cambio permanente, la innovación y el avance acelerado del conocimiento, la ciencia y la tecnología, las competencias para aprender a aprender, aprender haciendo, aprender a trabajar en equipo y aprender a lo largo de la vida resultan indispensables e inciden directamente en el crecimiento, desarrollo y progreso social (Stiglitz y Greenwald, 2014). Simultáneamente, la omnipresencia de las tecnologías y aplicaciones digitales en medio de las cuales convive el mundo educativo contemporáneo y que impactan sobre todo a las nuevas generaciones de estudiantes (Tondeur et al., 2017), nos ubican en un escenario en el que las potencialidades provistas por la tecnología pueden aprovecharse para mejorar y ampliar las posibilidades de aprendizaje de los estudiantes, sus competencias de pensamiento crítico, solución de problemas, colaboración, comunicación y aprendizaje autodirigido (Hewlett Foundation, 2017).

En este escenario, las demandas sobre la competencia digital de los profesores se complejizan, exigiendo nuevas aproximaciones para integrar efectivamente la tecnología en la educación (Instefjord y Munthe, 2017), entre 
ellas: la necesidad de una visión integradora de la pedagogía, la tecnología y los conocimientos (Fullan y Langworthy, 2014); la actualización del rol del profesor, de facilitador a activador de las competencias fundamentales, en busca de que los estudiantes puedan vivir integralmente como ciudadanos del siglo xxi (Hattie, 2012); el trabajo sobre las potencialidades y el sentido de uso de las tecnologías para promover ecologías de aprendizaje (Kalantzis y Cope, 2015); el aprendizaje a través de experiencias auténticas para reconocer las ecologías de trabajo en las escuelas y las posibilidades de integración e interconexión de las TIC con sujetos, contextos y saberes (Downes, 2012; Voogt, Fisser, Tondeur y van Braak, 2016). Esto constituye un importante desafío para la formación inicial de las próximas generaciones de profesores (Liu, 2016; Mouza, Karchmer-Klein, Nandakumar, Ozden y Hu, 2014; Tondeur et al., 2017).

\section{Aprovechar y promover los affordances de las TIC para la formación inicial de profesores}

El tercer gran interrogante de esta reflexión se enfoca en la forma en que pueden aprovecharse los affordances de las TIC para actualizar y hacer más pertinentes los procesos de formación inicial de profesores en el desarrollo de sus competencias para integrar las TIC en la educación. En este sentido, se propone que el desafío consiste en enfrentarse a la construcción de una metáfora ecosistémica, que se distancie de las tradicionales reflexiones e investigaciones educativas que oponen los ambientes físicos a los digitales, la educación presencial a la educación virtual, la pedagogía a la tecnología, y por el contrario, promueva la integración de estos escenarios educativos a partir de dos metáforas conceptuales: los affordances y las ecologías de aprendizaje.

Esta propuesta va de la mano con el análisis realizado a las disrupciones educativas creadas por el mundo interconectado, la omnipresencia de la información y la tecnología, las cuales han propiciado nuevas formas de representación y práctica del aprendizaje. Entre ellas: discontinuidades espacio-temporales; complejas interacciones entre sujetos, artefactos y ambientes; disponibilidad de variadas fuentes de información en múltiples formatos; coexistencia de escenarios físicos, virtuales, formales e informales; así como la dinámica en la que todos nos constituimos en aprendices permanentes y diseñadores activos de nuestras propias rutas de conocimiento. Estas disrupciones plantean retos que deben abordar los programas de formación inicial de profesores con el fin de hacer de sus estudiantes sujetos preparados para dialogar con los nuevos paradigmas educativos y agentes activos del desarrollo de las formas emergentes de aprendizaje (Leal-Urueña, 2017).

De acuerdo con Cope y Kalantzis (2017) el fenómeno denominado ecologías de aprendizaje recibe esta denominación debido a que los ambientes de aprendizaje son en cierto sentido como "ecosistemas caracterizados por la compleja, coherente y sistémica interacción de las dinámicas humana, textual, discursiva y 
espacial" (p. 1). En este sentido, de acuerdo con Maina y García (2016) tanto los contextos educativos formales como los informales hacen parte de las ecologías de aprendizaje; y tanto las instituciones, los docentes y el modelo pedagógico, como las prácticas, redes y relaciones de interdependencia desempeñan un papel fundamental en la estructuración de sus componentes.

De esta manera, y entendiendo que el ecosistema educativo contemporáneo es un universo interconectado en el que se integran diferentes sistemas de orden social, cultural, tecnológico, económico, político y territorial, alternos al sistema educativo formal y articulados con este de modo diverso y múltiple, en el cual la tecnología cada día tiene un papel más protagónico, surgen en esta integración los potenciales escenarios de aprendizaje o vectores fuerza del aprendizaje denominados affordances (Cope, 2016; Cope y Kalantzis, 2009).

Los affordances sintetizan el producto de la emergencia de procesos de conocimiento e innovación generados a partir de la continua interacción entre artefacto, sujeto y ambiente (Parchoma, 2014; Rojas-Mesa y Leal-Urueña, 2017). En este sentido, las ecologías de aprendizaje proveen múltiples affordances o potencialidades para transformar efectivamente la práctica pedagógica y por ende la formación inicial de profesores (Leal-Urueña, 2017). A continuación se describen seis affordances de la relación entre educación y tecnología que el docente puede tomar como ruta para la incorporación de nuevos sentidos del aprendizaje contemporáneo (véase la tabla 3).

Tabla 2. Affordances de la relación entre educación y tecnología: oportunidades para la formación inicial de profesores en competencias TIC

\begin{tabular}{|c|c|}
\hline Affordance & Descripción \\
\hline Ubicuidad & $\begin{array}{l}\text { Derivado de los avances de la computación ubicua y en la nube, ha dado origen a formas de aprendizaje } \\
\text { que exigen de los futuros profesores la capacidad para gestionar las discontinuidades espaciales y } \\
\text { temporales en las que se desarrollan los procesos educativos (Burbules, 2012). Potencia los entornos } \\
\text { personales de aprendizaje (PLE) y en red (PNL) (Kalantzisy Cope, 2015). }\end{array}$ \\
\hline $\begin{array}{l}\text { Crea } \\
\text { difusi }\end{array}$ & $\begin{array}{l}\text { Abren la posibilidad de convertir en públicos los artefactos generados durante el proceso formativo, } \\
\text { mediante su difusión y visibilización a través de las plataformas de redes sociales tales como blogs, wikis, } \\
\text { sitios para compartir video y aplicaciones web, entre otras (Burbules, 2009). }\end{array}$ \\
\hline Evalua & $\begin{array}{l}\text { Las tecnologías disponibles permiten desarrollar procesos de evaluación más flexibles, participativos y } \\
\text { recursivos para proporcionar retroalimentación continua, constructiva y prospectiva (Smith et al., 2017). }\end{array}$ \\
\hline $\begin{array}{l}\text { Trabajo } \\
\text { colaborativo }\end{array}$ & $\begin{array}{l}\text { Las actividades colaborativas de carácter presencial, virtual o mixto se pueden estructurar mediante } \\
\text { diferentes tipos de scripts que definen la tarea por desarrollar, la composición del grupo, la forma en } \\
\text { que la tarea se distribuye dentro y entre los grupos, el modo de interacción y el tiempo de cada fase de } \\
\text { trabajo (Stahl, 2014; Stahl, Koschmann y Suthers, 2015). }\end{array}$ \\
\hline Metacognición & $\begin{array}{l}\text { En el desarrollo de las capacidades de monitoreo y regulación de la práctica pedagógica del profesor en } \\
\text { formación se pueden integrar diversas tecnologías para recolectar sus registros de observación, analizar } \\
\text { las conductas de los estudiantes en búsqueda de patrones de aprendizaje, y elaborar inferencias y } \\
\text { explicaciones sobre el proceso de aprendizaje en función de su modelo de clase (Sanabria, Vargas y Leal, } \\
\text { 2014). }\end{array}$ \\
\hline Multimodalidad & $\begin{array}{l}\text { Útil para desarrollar las actividades de aprendizaje que resultan difíciles o imposibles de implementar sin } \\
\text { el soporte tecnológico adecuado, tales como representar adecuadamente los conceptos que sustentan } \\
\text { algunos dominios de conocimiento, su aplicación, prueba de hipótesis, simulaciones, toma de decisiones } \\
\text { complejas, modelamiento, comunicación y colaboración con expertos, aprendizaje personalizado y } \\
\text { adaptativo, etc. (Angeli, Valanides y Christodoulou, 2017). }\end{array}$ \\
\hline
\end{tabular}

Fuente: elaboración propia. 
Poner en marcha los procesos de formación inicial de profesores a través de las perspectivas que plantean las ecologías de aprendizaje y los affordances de las TIC facilitaría, entre otros, el desarrollo de la inteligencia colaborativa para afrontar los desafíos de la transferencia, adaptación e integración de tecnología; la construcción de escenarios de aprendizaje más interesantes y conectados con la realidad; ricas experiencias de incorporación de tecnología e innovación de las prácticas pedagógicas mediante la retroalimentación entre pares; el uso e intercambio de materiales en multiformato para construir múltiples formas de representación del conocimiento, así como la flexibilización y adaptación del proceso de aprendizaje, de acuerdo con los intereses de los estudiantes.

\section{Conclusiones}

A pesar de todo el conocimiento disponible, estudios recientes informan que el uso de la tecnología y el desarrollo de la competencia digital durante la formación inicial de los profesores continúan siendo insuficientes. Además indican que existe un desequilibrio entre las competencias digitales que requieren los profesores para desempeñarse profesionalmente al integrar la tecnología con propósitos educativos y las que desarrollan durante su proceso formativo (Gudmundsdottir, Loftagarden y Ottestad, 2014). Sobre este vacío no se identifica el liderazgo de las instituciones educativas en la producción de lineamientos o planes para tratar el problema.

Dentro de las recomendaciones realizadas con mayor frecuencia en la literatura especializada se encuentran la de acompañar la integración de tecnología con una renovación de las prácticas pedagógicas (Kalantzis y Cope, 2015) y de los diseños curriculares (Agyei y Voogt, 2014) para hacerlos acordes a los desafíos de la producción de conocimiento y las nuevas formas de aprendizaje.

Las estrategias de aprendizaje analizadas en esta reflexión, cuya efectividad se ha evidenciado a partir de diversas investigaciones, representan un importante recurso para reformar las prácticas a través de las cuales se desarrolla la formación inicial de profesores. Estas estrategias constituyen una primera aproximación articuladora de las prácticas pedagógicas, el aprendizaje y la tecnología y evidencian las potencialidades de las TIC para el desarrollo de los procesos educativos.

En este escenario, se hace muy importante construir una perspectiva ecosistémica de los problemas educativos contemporáneos, que hable de su integración con sistemas culturales, sociales, tecnológicos, entre otros, y no de su independencia u oposición como ha ocurrido hasta ahora. Aspectos tan relevantes como la posibilidad de integrar multiformatos o combinar espacios de aula con entornos digitales y escenarios de formación formal e informal, y la ruptura de los cánones clásicos de uso del espacio y del tiempo son algunas características de las nuevas ecologías de aprendizaje. 
Los affordances permiten construir un lugar para el artefacto sin clasificarlo en su naturaleza física o digital, sino más bien en la transición entre ellas. Asimismo, dan sentido a la emergencia y necesaria renovación del artefacto como producto de procesos de aprendizaje y producción de saber. Los affordances de ubicuidad, creación y difusión, evaluación, trabajo colaborativo, metacognición y multimodalidad propuestos en esta reflexión fortalecen las estrategias de integración de las TIC en la educación y los escenarios de formación docente.

Las TIC proporcionan diversas rutas para implementar procesos de formación inicial de educadores, y crean escenarios para la vivencia y comunicación del docente con las nuevas generaciones de estudiantes. Así potencian diversas formas de aprendizaje y producción de conocimiento en una era de ecosistemas que integran entornos físicos y digitales.

\section{Referencias}

Agyei, D. D., y Voogt, J. M. (2014). Examining factors affecting beginning teachers' transfer of learning of ict-enhanced learning activities in their teaching practice. Australasian Journal of Educational Technology, 30(1), 92-105. doi: https://doi.org/10.14742/ajet.499

Angeli, C., Valanides, N. y Christodoulou, A. (2017). Theoretical considerations of Technological Pedagogical Content Knowledge. En M. Herring, M. J. Koehler y P. Mishra (eds.), Handbook of Technological Pedagogical Content Knowledge (tpack) for Educators (pp. 11 -32). Nueva York: Routledge.

Brun, M. (2011). Las tecnologías de la información y la comunicación en la formación inicial docente de América Latina. Santiago de Chile: Naciones Unidas. Recuperado de https://goo.gl/6zLzqc
Burbules, N. (2009). Meanings of "ubiquitous learning". En B. Cope y M. Kalantzis (eds.), Ubiquitous learning (pp. 15-20). Urbana (Champaign): University of Illinois Press.

Burbules, N. (2012). Ubiquitous Learning and the Future of Teaching. Encuentros, 3-14.

Cepal. (2007). elAC 2007 Agenda digital para América Latina y el Caribe. Recuperado de https://bit.ly/2mVJkf7

Cepal. (2010). Plan de acción sobre la sociedad de la información y del conocimiento en América Latina y el Caribe (elAC 2015). Recuperado de https://bit.ly/2NW9YQh

Cepal. (2015). Agenda digital para América Latina y el Caribe (elAC 2018). Recuperado de https://bit.ly/2LQn1C3

Cepal. (2018). elAC 2018 Agenda digital para América Latina y el Caribe (elAC 2020). Recuperado de https://bit.ly/2m499cg

Chien, Y., Chang, C., Yeh, T. y Chang, K. (2012). Engaging pre-service science teachers to act as active designers of technology integration: A Magdaire framework. Teaching and Teacher Education, 28, 578-588. doi:10.1016/i. tate.2011.12.005

Congreso de la República de Colombia. (1994). Ley general de educación. Bogotá: Ministerio de Educación Nacional.

Cope, B. (2016). Publications. Illinois College of Education. Recuperado de http://education. illinois.edu/faculty/billcope

Cope, B. y Kalantzis, M. (2017). e-Learning ecologies: Principles for new learning and assessment. Nueva York: Routledge.

Cope, W. y Kalantzis, M. (2009). Ubiquitous learning. Exploring the anywhere/anytime possibilities for learning in the age of the digital media. Urbana-Champaign: University of Illinois Press. 
Departamento Nacional de Planeación (DNP). (2015). Plan Nacional de Desarrollo 2014-2018 "Todos por un nuevo país". Recuperado de https://goo.gl/kiCHi8

Downes, S. (2012). Knowledge, learning and community. Recuperado de http://www. downes.ca/post/57737

Fullan, M. y Langworthy, M. (2014). Una rica veta. Cómo las nuevas pedagogías logran el aprendizaje en profundidad. Pearson. Recuperado de https://goo. gl/4MjTfV

Galvis, Á. (2014). Las políticas TıC en los sistemas educativos de América Latina: caso Colombia. Buenos Aires: Unicef.

Gudmundsdottir, G. B., Loftagarden, M. y Ottestad, G. (2014). Newly qualifed teachers. Professional digital competence and experiences with ict in teacher education. 17th Unesco-apeid International Conference: The Powerhouses of Education: Teachers for the Future We Want. Oslo. Recuperado de https://goo.gl/TEJTZ9

Hattie, J. (2012). Visible learning for teachers: Maximizing impact on learning. Nueva York: Routledge.

Hewlett Foundation. (2017). Hewlett Foundation. Recuperado de https://goo.gl/ $\mathrm{mCh} 6 \mathrm{dt}$

Instefjord, E. J. y Munthe, E. (2017). Educating digitally competent teachers: A study of integration of professional digital competence in teacher education. Teaching and Teacher Education, 67, 37-45. doi:http://dx.doi.org/10.1016/i. tate.2017.05.016

Intef. (2017). Marco común de competencia digital docente. Madrid: Gobierno de España. Ministerio de Educación, Cultura y Deporte.

ISTE. (2016). ISTE Standards for educators. USA: ISTE. Recuperado de https://goo.gl/ykfwuy

Kafai, Y. (1995). Minds in play: Computer game design as a context for children's learning. Mahwah, NJ, us: Lawrence Erlbaum.

Kalantzis, M. y Cope, B. (2015). Learning and new media. En D. Scott y E. Hargreaves (eds.), The Sage handbook of learning (pp. 373-387). Londres: sage Publications. doi:http://dx.doi.org/10.4135/9781473915213

Kali, Y., McKenney, S. y Sage, O. (2015). Teachers as designers of technology enhanced learning. Instructional Science, 43(2), 173-179. doi:10.1007/s1 1251014-9343-4

Leal-Urueña, L. A. (2017). Potencialidades de las ecologías de aprendizaje para la formación inicial de profesores en integración de tecnologías en la educación. Actas del iii Congreso Internacional de Educación Mediática y Competencia Digital (pp. 2627-2639). Segovia (España): Universidad de Valladolid.

Li, Q. (2012). Understanding enactivism: A study of affordances and constraints of engaging practicing teachers as digital game designers. Educational Technology Research and Development, 785-806. 
Ling, J. y Chai, C. (2016). Seven design frames that teachers use when considering technological pedagogical content knowledge (tpack). Computers \& Education, 102, 244 257. doi:10.1016/j.compedu.2016.09.003

Liv, S. H. (2016). Teacher education programs, field-based practicums, and psychological factors of the implementation of technology by pre-service teachers. Australasian Journal of Educational Technology, 32(3), 65-79. doi:https://doi.org/10.14742/ajet.2139

Maina, M. y Garcia, I. (2016). Articulating personal pedagogies through learning ecologies. En B. Gros, Kinshuk y M. Maina (eds.), The future of ubiquitous learning: Learning designs for emerging pedagogies (pp. 73-94). Berlín: Springer. doi: 10.1007/978-3-662-47724-3

McKenney, S., Kali, Y., Markauskaite, L. y Voogt, J. (2015). Teacher design knowledge for technology enhanced learning: An ecological framework for investigating assets and needs. Instructional Science, 43(2), 181-202. doi:10.1007/s11251-014-9337-2

Ministerio de Comunicaciones. (2008). Plan Nacional de tic 2008-2019. Todos los colombianos conectados, todos los colombianos informados. Recuperado de https://goo.gl/ MwuxBp

Ministerio de Educación Nacional (MEN). (2009). Decreto 5012 de 2009. Recuperado de https://goo.gl/xzzy2p

Ministerio de Educación Nacional (MEN). (2013). Competencias TIC para el desarrollo profesional docente. Bogotá: Imprenta Nacional de Colombia. Recuperado de https://goo. $\mathrm{gl} / \mathrm{ewpfrl}$

Ministerio de Educación Nacional (MEN). (2017a). Plan Nacional Decenal de Educación 2016 2026. El camino hacia la calidad y la equidad. Bogotá: autor. Recuperado de https:// goo.gl/4cYjigR
Ministerio de Educación Nacional (MEN). (2017b). Resolución 18583 del 15 de septiembre de 2017, por la cual se ajustan las características específicas de calidad de los programas de licenciatura para la obtención, renovación o modificación del registro calificado. Bogotá, Colombia.

Mouza, C., Karchmer-Klein, R., Nandakumar, R., Ozden, S. Y., y Hu, L. (2014). Investigating the impact of an integrated approach to the development of preservice teachers' technological pedagogical content knowledge (tpack). Computers \& Education, 71 , 206-221. doi:https://doi.org/10.1016/i. compedu.2013.09.020

Niess, M. L. (2017). Transforming teachers' knowledge for teaching with technologies: An online learning trajectory instructional approach. En M. Herring, M. J. Koehler y P. Mishra (eds.), Handbook of technological pedagogical content knowledge (tpack) for educators (pp. 131-142). Nueva York: Routledge.

Olsson, L. y Edman-Stålbrant, E. (2008). Digital literacy as a challenge for teacher education. En M. Kendall y B. Samways, Learning to live in the knowledge society (pp. 11-18). Boston: Springer.

Orealc/Unesco, S. (2016). Tecnologías digitales al servicio de la calidad educativa. Una propuesta de cambio centrada en el aprendizaje para todos. Santiago: Unesco.

Organización de Estados Iberoamericanos (OEI). (2010). 2021 metas educativas. La educación que queremos para la generación de los bicentenarios. Madrid: OEl y Cepal.

Paavola, S. y Hakkarainen, K. (2009). From meaning making to joint construction of knowledge practices and artefacts: A trialogical approach to CSCL. Computer Supported Collaborative Learning Practices: CsCL2009 
conference proceedings (pp. 83-92). Rhodes: International Society of the Learning Sciences (ISLS).

Papert, S. (1980). Mindstorms: Children, computers, and powerful ideas. Nueva York: Basic Books.

Parchoma, G. (2014). The contested ontology of affordances: Implications for researching technological affordances for collaborative knowledge production. Elsevier Computers in Human Behavior, 360-368.

Rojas-Mesa, J. y Leal-Urueña, L. (2017). Affordance: Constructo para la comprensión y transformación del aprendizaje en contextos interculturales. Tecné, Episteme y Didaxis: TED, 42. Recuperado de https://goo.gl/nxxrts

Sanabria, L., Vargas, O. y Leal, L. (2014). metacognitive and investigative skill development in pre-service teachers through the use of digital technologies-contributions to teaching excellence. Revista Colombiana de Educación, 67, 147-170.

Scardamalia, M. y Bereiter, C. (2014). Knowledge building and knowledge creation: Theory, pedagogy and technology. En K. S. (ed.), Cambridge handbook of the learning sciences (2.a ed.). Cambridge, UK: Cambridge University Press.

Siteal. (2014). Políticas tıc en los sistemas educativo de América Latina. Paris, Buenos Aires, Madrid: Unesco-IIPE-OEI.

Smith, A., McCarthey, S. y Magnifico, A. (2017). Recursive feedback. Evaluative dimensions of e-Learning. En B. Cope y M. Kalantzis (eds.), e-Learning ecologies: Principles for new learning and assessment (pp. 1 18-142). Nueva York: Routledge.

Stahl, G. (2014). The constitution of group cognition. En L. Shapiro (ed.), Handbook of embodied cognition. Nueva York: Routledge.

Stahl, G., Koschmann, T. y Suthers, D. (2015). Computer-supported collaborative learning: An historical perspective. En R. K. Sawyer (ed.), Cambridge handbook of the learning sciences. Cambridge, UK: Cambridge University Press.

Stiglitz, J. y Greenwald, B. (2014). Creating a learning society: A new approach to growth, development and social progress. Nueva York: Columbia University Press.

Tondeur, J., Braak, J., Sang, G., Voogt, J., Fisser, P. y Ottenbreit-Leftwich, A. (2012). Preparing pre-service teacher to integrate technology in education: A synthesis of qualitative evidence. Computers \& Education, 134-144.

Tondeur, J., Pareja Roblin, N., van Braak, J., Voogt, J. y Prestridge, S. (2017). Preparing beginning teachers for technology integration in education: Ready for take-off? Technology, Pedagogy and Education, 26(2), 157-177. doi:10.1080/147593 9X.2016.1193556

Tondeur, J., Scherer, R., Siddiq, F. y Baran, E. (2017). A comprehensive investigation of tpack within pre-service eachers' ICT profiles: Mind the gap! Australasian Journal of Educational Technology, 33(3), 46-60. doi:10.14742/ajet.3504 
Unesco. (201 1). ICT Competency Framework for Teachers. Paris: United Nations Educational, Scientific and Cultural Organization.

Vaillant, D. (2013). Integración de TIC en los sitemas de formación docente inicial y continua para la Educación Básica en América Latina. Buenos Aires: Unicef.

Voogt, J., Fisser, P., Tondeur, J. y van Braak, J. (2016). Using theoretical perspectives in developing understanding of tpack. En M. C. Herring, M. J. Koehler y P. Mishra (eds.), Handbook of technological pedagogical and content knowledge for educators (pp. 33-52). Nueva York: Routledge.

Wilson, C., Grizzle, A., Tuazo, R., Akyempong, K. y Cheung, Ch. (2011). Alfabetización mediática e informacional. Currículum para profesores. París: Unesco.

\section{Para citar este artículo}

Leal-Urueña, L. y Rojas-Mesa, J. (2018). Ecología para la formación inicial de profesores a partir de los affordances de las TIC. Tecné, Episteme y Didaxis: TED, $44,15-31$ 